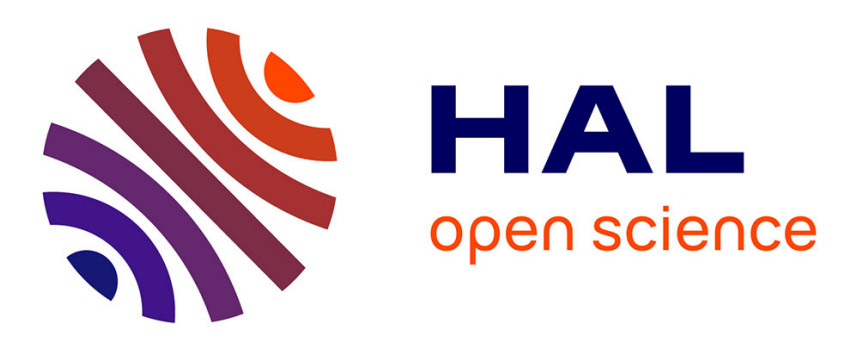

\title{
Diversifying the picture of explanations in biological sciences: ways of combining topology with mechanisms \\ Philippe Huneman
}

\section{To cite this version:}

Philippe Huneman. Diversifying the picture of explanations in biological sciences: ways of combining topology with mechanisms. Synthese, 2018, 195 (1), pp.115-146. 10.1007/s11229-015-0808-z . hal01968466

\section{HAL Id: hal-01968466 https://hal.science/hal-01968466}

Submitted on 10 Jan 2019

HAL is a multi-disciplinary open access archive for the deposit and dissemination of scientific research documents, whether they are published or not. The documents may come from teaching and research institutions in France or abroad, or from public or private research centers.
L'archive ouverte pluridisciplinaire HAL, est destinée au dépôt et à la diffusion de documents scientifiques de niveau recherche, publiés ou non, émanant des établissements d'enseignement et de recherche français ou étrangers, des laboratoires publics ou privés. 


\section{Diversifying the picture of explanations in biological sciences: ways of combining topology with mechanisms}

\section{Philippe Huneman}

\section{Synthese}

An International Journal for Epistemology, Methodology and Philosophy of Science

ISSN 0039-7857

Synthese

DOI 10.1007/s11229-015-0808-z

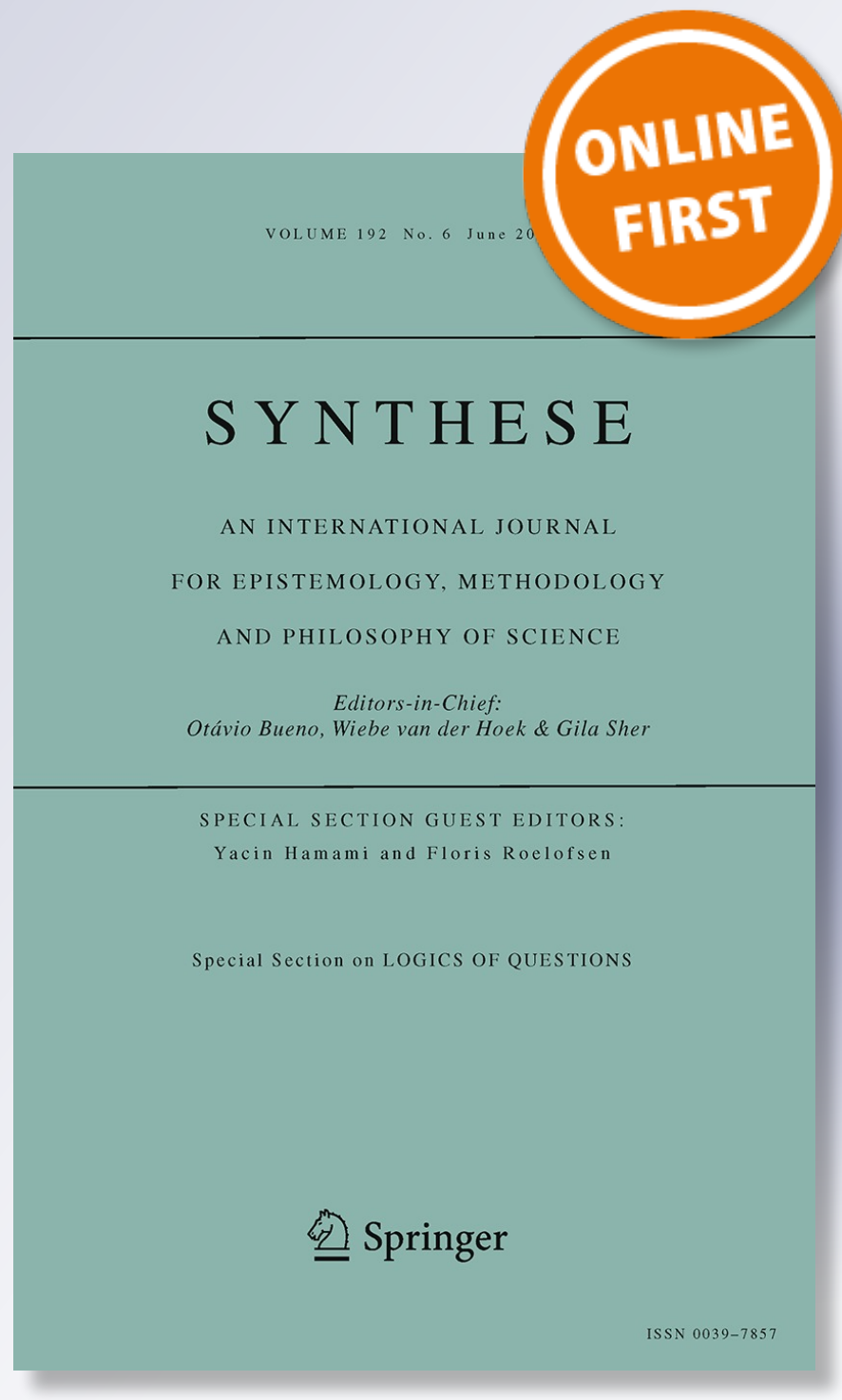

黛 Springer 
Your article is protected by copyright and all rights are held exclusively by Springer Science +Business Media Dordrecht. This e-offprint is for personal use only and shall not be selfarchived in electronic repositories. If you wish to self-archive your article, please use the accepted manuscript version for posting on your own website. You may further deposit the accepted manuscript version in any repository, provided it is only made publicly available 12 months after official publication or later and provided acknowledgement is given to the original source of publication and a link is inserted to the published article on Springer's website. The link must be accompanied by the following text: "The final publication is available at link.springer.com". 


\title{
Diversifying the picture of explanations in biological sciences: ways of combining topology with mechanisms
}

\author{
Philippe Huneman ${ }^{1}$
}

Received: 4 December 2014 / Accepted: 21 June 2015

(C) Springer Science+Business Media Dordrecht 2015

\begin{abstract}
Besides mechanistic explanations of phenomena, which have been seriously investigated in the last decade, biology and ecology also include explanations that pinpoint specific mathematical properties as explanatory of the explanandum under focus. Among these structural explanations, one finds topological explanations, and recent science pervasively relies on them. This reliance is especially due to the necessity to model large sets of data with no practical possibility to track the proper activities of all the numerous entities. The paper first defines topological explanations and then explains why topological explanations and mechanisms are different in principle. Then it shows that they are pervasive both in the study of networks - whose importance has been increasingly acknowledged at each level of the biological hierarchy-and in contexts where the notion of selective neutrality is crucial; this allows me to capture the difference between mechanisms and topological explanations in terms of practical modelling practices. The rest of the paper investigates how in practice mechanisms and topologies are combined. They may be articulated in theoretical structures and explanatory strategies, first through a relation of constraint, second in interlevel theories (Sect. 3), or they may condition each other (Sect. 4). Finally, I explore how a particular model can integrate mechanistic informations, by focusing on the recent practice of merging networks in ecology and its consequences upon multiscale modelling (Sect. 5).
\end{abstract}

Keywords Topological explanation $\cdot$ Networks $\cdot$ Community ecology $\cdot$ Mechanism . Population genetics · Evolution

Philippe Huneman

Philippe.huneman@gmail.com

1 IHPST (CNRS/Université Paris 1 Sorbonne), 13 rue du Four, 7506 Paris, France

Published online: 10 July 2015 


\section{Introduction}

It has been successfully argued for more than a decade that many biological explanations proceed by uncovering mechanisms. Although what "mechanisms" are differ according to differing accounts (e.g. Glennan 1996, Bechtel and Abrahamsen 2005, Craver and Darden 2013), all of these accounts concur in saying that mechanisms consist in a set of entities with proper activities, whose joint outcomes in a specific setting yield the phenomenon to be explained. Molecular biology provides many examples of such explanations, as can be argued by considering the case of lactose operon studied by Jacob and Monod, or allosteric interactions in protein synthesis (Monod et al. 1965). If we follow Craver (2007) highly convincing account, neurosciences also seem to share the same explanatory regime, for instance when researchers study the features of ion channels involved in memory phenomena.

Even though "mechanism" has a long tradition in philosophy, tracing back at least to Descartes, the meaning of this concept among those who came to be known as "the new mechanicists" seems to focus on two points: the idea that an explanation of the system has to rest on the explanation of the parts-i.e. Kant's idea of mechanism, but tempered by the acknowledgement that mechanisms have to be understood in their global context (e.g. Craver 2013); and the intent to capture what scientists mean when they write, for example, "the mechanism of alarm call emitting", or "the mechanism of sex addiction". However, although such uses of the term "mechanism" are usually understood by other scientists, the speaker may not intend to convey a precise or technical meaning; the expression is often simply understood to be synonymous with "explanation of", and taken in opposition with the notion of "phenomenological model", which consists in mathematically capturing a pattern present in the data.

Yet it is doubtful whether all explanations are mechanistic in the ways just described, and it has been argued that some explanations in biology are instead non-mechanistic (Huneman 2010a; Woodward 2013; Jones 2014; Rice 2012) or perhaps even noncausal (Baker 2009; Batterman and Rice 2014). According to this perspective, biology and especially evolutionary biology and ecology are characterized by a pluralism regarding explanation, in the sense that distinct explanatory modes coexist in those fields. This view holds that in some of these modes detailed mechanisms are not explanatory as such, that mathematical features instead play a key explanatory role. I will call them "structural explanations", meaning that in these explanations structures of any mathematical type do the explanatory job.

Structural explanations come in many kinds. Optimality reasoning (Maynard-Smith 1980 for behavioural ecology) is one of them, in the sense that the mere fact that a state is optimal-which is something mathematically determined in terms of extrema of some function, even though the model itself is built upon empirical observations - is seen as a candidate explanation that it is approximately met in nature, ${ }^{1}$ notwithstanding the various processes that may bring it about. Microeconomics is full of explanations that do not consider mechanisms in the sense of the various activities possibly undergone by various agents. In other contexts, the central limit theorem, which states that

\footnotetext{
${ }^{1}$ See Potochnik (2009) and Rose and Lauder (1996) on optimisation in behavioural ecology.
} 
an addition of independent random variables with the same variance and mean necessarily gives a normal distribution (conditions can even be relaxed but it does not matter here), and affords us with an explanation of many facts such as all the normal patterns we find in nature (Frank 2009; see Lyon 2013 for a critique), grounds instances of structural explanations. Although explanatory, it does not hinge upon the various mechanisms which support each of the added random variables. However, in this paper I focus on the explanations relying on properties that are topological in a very broad sense (Huneman 2010a; Woodward 2013; Jones 2014), which includes graph theory.

Given the acknowledgement of the coexistence of topological explanations and mechanistic explanations in biology and ecology, this paper questions the ways they are different and can relate to each other, both in the general structure of a given discipline, and within one explanatory or modelling strategy. The first section defines topological explanations and questions how topological explanations are in principle different from mechanistic explanations, discussing a view that situates both of them within a continuum of increasing abstraction. The second section identifies several places in contemporary biological science where they are found-making a case that the role of these explanations is increasingly important in science-and argues that for reasons regarding this scientific practice one should consider topological explanations apart from mechanisms. Then I will study how topologies and mechanisms can be articulated with one another in the architecture of theories: the following section considers the constraining impact of topologies upon mechanisms and their articulation within interlevel theories, while section four examines the relation of conditioning that may hold between topologies and mechanisms in scientific theories. The last section considers the integration of these two explanatory modes within single models, focusing on recent work that uses network analysis in ecology. Articulation and integration of topological and mechanistic explanation thereby provide a pluralistic picture of explanatory practices in biology and ecology.

\section{Topological explanations: notion, and distinctions}

\subsection{The notion of topological explanation}

Formally speaking, a topological explanation is an explanation in which a feature, a trait, a property or an outcome $\mathrm{X}$ of a system $\mathrm{S}$ is explained by the fact that it possesses specific topological properties $\mathrm{T}_{\mathrm{i}}$. "Possessing topological properties" can be explicated as follows:

- a system $S$ under focus is related to a topological space S', which can be the actual space where the parts of the system itself exist, or often a more abstract space like a phase space, a network of relations or interactions expressed by a graph, etc.; in this space can be included parts of the system, behaviors of the system, capacities, or any other features correlated to the system (the trajectory, some interactions, etc.) (Examples of such space would be: the trophic networks in theoretical ecology, the networks of habitats in metapopulation ecology (Hanski 1998), or the phase space of mechanical or statistical-mechanics systems); 
- topological properties are properties of $\mathrm{S}^{2}$ that specify its invariance regarding a class of continuous transformations. (For instance compactness or connexity are topological properties to the extent that no continuous transformation can transform a connex set into a non-connex set, or a compact into a non-compact set.) Consequently, for any set $\mathrm{X}$ of continuous transformations, topological properties define equivalence classes $C_{X}$, namely, classes of manifolds that are equivalent regarding $X$, i.e. each of them being the transform of another through a function that belongs to $\mathrm{X}$.

In this context, I extend "topological" to graph-theoretical properties, according to the intention of the recent "topological graph theory" (Gross and Tucker 1987). In effect, a combination of graph-theoretical properties (such as a connectance value, cyclicity, a clustering coefficient (Strogatz 2001; Lesne 2007)—see Box 1 for definitions of technical terms) also defines equivalence classes between graphs, in the sense that the graphs sharing these properties are such that for a large set of transformations $\mathrm{X}$ they are equivalent. This in turn defines ipso facto some kinds of invariance; e.g., a network in the class "scale-free networks" will remain scale-free by deleting some nodes or switching some connections, hence remain in the same class. ${ }^{3}$

\section{Box 1. Some terms in network theory.}

Degree (of a vertex): number of edges incident to a given vertex.

Cycle: sequence of vertices with each two consecutive vertices in the sequence adjacent to each other in the graph, and no repetition of edges or vertices except the final and initial vertex.

Local clustering coefficient $c$ : taking all the neighbors of a vertex $\mathrm{V}, \mathrm{c}$ is the proportion of links between the vertices within its neighbourhood divided by the number of links that could possibly exist between them (it quantifies how close the neighborhood of $\mathrm{V}$ is a network is to being a "clique", i.e. a wholly connected network).

Average clustering coefficient: average of local coefficient of all vertices.

Connectance (in ecology): the proportion of possible links between species that are realized (links/species ${ }^{2}$ ).

Path: finite or infinite sequence of vertices (the number of vertices in the shortest path between two vertices is their distance).

Scale-free network: network whose degree distribution follows a power law (from very few hubs highly connected, to very large amount of nodes with a single connection; airlines maps are an example).

Small world network: network which has a high clustering coefficient but a small average distance between nodes (in contrast, random graphs have a small average distance and a small clustering coefficient). This property provides them with a kind of modularity.

Preferential attachment: network building process rule in which the probability for an entity (a node) of having a new connection is proportional to the amount of connections it already has. Sometimes called "rich get richer" rule.

\footnotetext{
2 One is however entitled to say that these properties are secondarily properties of the system itself. Think of properties of a food web of an ecological community, for instance: such a property can legitimately be seen as a property of the community itself.

${ }^{3}$ In this context one can of course recall that historically the same problem was at the source of topology and of graph theory-i.e. the "bridges of Königsberg" problem, namely, the problem of knowing whether there exists one pathway through which a traveller can cross all the seven bridges of Königsberg just once. Euler solved it, laying the bases of topology and later graph theory. The answer is negative. All the graphs with seven "bridges" (i.e. "edges" in graph theory) that are such that no such pathway exists are therefore equivalent regarding any permutation of vertices — and the same thing holds for all networks that allow one pathway with exactly one double crossing, etc.
} 
Whenever the explanandum - a property, outcome, behavior of S-is explained by the fact that the system has topological properties $\mathrm{T}_{\mathrm{i}}$, I thereby say that a topological explanation has been given. "Explanations" here means that some fact $\mathrm{G}$ is entailed by the topological properties $T_{i}$, and is itself a mathematical fact that describes adequately the explanandum under focus. Some examples of topological explanations include the following: Jones (2014) has shown that pinpointing the bow-tie structure of interactions in immune defense as an explanation of the vulnerability on attacks on CD4+ T-cells is a straightforward topological explanation. The vulnerability under focus is mathematically expressed in terms of a higher probability proper to attacks on these cells as compared to attacks onto other cells; which is in turn entailed by the bow-tie structure especially uncovered by Kitano and Oda (2006). Huneman (2010a) argued that explaining the stability of an ecological community by alluding to some formal character of the trophic network or interaction network-for instance, that it is a scale-free network - constitutes a topological explanation. In this latter example the scale-freeness (or truncated scale-freeness, more generally) entails that the probability of altering the existence of the network by randomly deleting some species is extremely low, which describes in mathematical terms a type of stability of the system.

\subsection{Distinction between kinds of explanation, with a focus on topological explanations}

Thus, in topological explanations, the topological facts are explanatory, and not the various processes that in nature instantiate variously these properties. This distinguishes them from mechanistic explanations, conceived in the sense of "new mechanicism", i.e. as the establishing of a set of entities with proper activities, organized in a specific ways, and whose joint outcome is the explanandum. But in topological explanations the processes that are going on in the systems under focus are involved in the explanation of the explanandum only in virtue of the topological facts that we uncover: for instance, in Jones' example above, all the biochemical interactions that preferentially lead to decrease in frequency of CD4+ T-cells have this outcome precisely because of the bow-tie structure and its consequences; in the second example mentioned all the possible ecological interactions yield a pattern of species stability only in so far as they instantiate the specific topology of networks we held responsible for it.

The crucial notion here is the difference between a descriptive or representational role of mathematics, and an explanatory role of it-mathematics being here the topology (broadly construed). For all mechanisms, of course current science use mathematics to model them, characterizing mathematically the "activities" at stake, often in the form of differential equations, transition probabilities, or any other mathematical tool. This is representational: natural language is not suited to precisely characterize these activities while mathematics are a better language regarding precision and abstraction, therefore we use them to describe what happens and, most noticeably, to predict outcomes (and therefore test and validate the models through a type of hypothetico-deductive model testing). But here the entities' activities themselves are explanatory, not the mathematics that describe them. Yet what happens with structural explanations in general is that mathematics are rather explanatory: 
mathematical properties are the reason why some facts happen in nature as outcomes or features of the activities of the hypothesised entities. The mathematics not only represent the mechanisms' settings and functioning, they also explain why a set of mechanisms is constrained in a specific way, necessarily yielding a range of outcomes that possess a given property. This includes a weak, epistemic claim, stating that without the mathematics we would never know that some outcomes are possible, some impossible and some necessary. But it also means the stronger, metaphysical, claim that in some cases the reason why some systems are displaying a constant or regular behavior of some sort (e.g., with a specific steady state, a typical outcome, or inversely, an absence of some particular outcome etc.) is a mathematical-in the present context, topological-fact: such fact grounds counterfactual dependences between sets of possible initial states and sets of end-states. Correlatively, this implies that what makes a difference regarding several sets of systems - for instance, stable and instable communities in the ecological example above-is a topological property; such property is instantiated by all mechanisms in the considered systems, but it's only in virtue of the fact that they instantiate this property that those are themselves explanatory of anything. Topology being about invariance through a class of continuous transformations, topological explanations are explanations in which the possibility and impossibility of some systems to reach some sets of final states or behaviors is explained by the topological fact which they instantiate, specifying which states are topologically equivalent and which are not, hence are not likely to be reached by the system.

This difference (between representational and explanatory status) should not be confused with an important distinction used by scientists to characterize their models, and which is in some sense involved in the concept of "mechanism" put forth by the new mechanicists - namely, the distinction between "phenomenological" and "mechanistic" model (or, in some other places like often evolutionary biology, the distinction between "model of pattern" and "model of process"). A "phenomenological model" mathematically represents the data, according to some requisites of simplicity and predictability that involve sophisticated statistical considerations. A "mechanistic model" explains why this pattern of data holds or should hold, based on hypotheses about the nature of the system. For example, showing that the relationship between area of ecosystems and number of species included obeys a mathematical law is a pattern model; a mechanistic model shows that specific processes of competition and dispersal are likely to yield such a law. Scientific methodologies often favor mechanistic models, or more precisely, search for mechanistic models once they have phenomenological models - in other words, search the processes when they have the patterns.

I claim that this distinction, often operational for scientists, is orthogonal to the abovementioned distinction between "mechanistic" in the sense of mechanicist philosophers and structural or topological explanations. Stated like this it may seem obvious but there is a natural tendency to assimilate these two distinctions, because a phenomenological or pattern model is indeed a mathematical formulation that does not hypothesize mechanisms, and because "mechanistic models" are commonly supposed to be more explanatory (phenomenological models merely represent patterns; according to the old adage, they "save the phenomena" instead of explaining them). Notice that "mechanistic" in these two distinctions is therefore not meaning the same thing-mechanistic sensu the scientists means "explaining a pattern" and mechanis- 
tic sensu neo-mechanistic philosophers means "explanation fulfilling their specific view of mechanisms"- - so that there is no vicious circularity in decoupling the two distinctions. $^{4}$

Kaplan and Craver (2011) indeed provide a defense of mechanicism against the claim that "dynamical models" (in the sense of models of systems based on nonlinear dynamics), which are non-mechanistic models, are the proper explanations for neuroscience (see e.g. Van Gelder 1995). They show that the dynamical equations representing the in-phase motion of opposite arms are not a mechanistic model, ${ }^{5}$ but a phenomenological model, which is perfectly true. A mechanistic model would look for the reasons why a pattern of motion that obeys such law is indeed produced by cognitive systems. They reaffirm here the well established idea that scientists should favor mechanistic models: "providing a general description of a phenomenon does not explain that phenomenon. Asked why the neuronal membrane changes its conductance to sodium and potassium ions as it does, it does not help to respond that all (properly functioning) neuronal membranes do so as well" (p. 622). But in my viewpoint they conflate the "mechanistic model" in the sense of scientists-i.e., a model that accounts for the pattern model by hypothesizing something about the system-and "mechanistic explanation" in the sense of "new mechanicism"-i.e. a model that is explanatory in virtue of the fact that it displays mechanisms in the sense of mechanicists. Granted, they agree that "mechanistic model" may also include some mathematical formulations (it almost always happens!), ${ }^{6}$ but this is the representational role of mathematics.

Following Kaplan and Craver, taking a model like the dynamic model of in-phase motion in cognitive science or the species-area law in ecology as an explanation would be to conflate prediction and explanation, and therefore suffer from the same flaws "predictivists" like Hempel have met, those flaws that were later pinpointed by Salmon among others. However the fact that pattern models in principle are written in mathematical terms (to put it bluntly, they are about curve fitting) in a way that mathematics does all the predictive job will not at all entail that "mechanistic models" should include mathematics only as representational and not as explanatory. Actually, the examples of topological explanations given above are "mechanistic models" since they explain why some patterns (stability of some communities, statistical repartition of various attacks on CD4+ T-cells) do occur. One should not confuse the equations of pattern models-which are indeed representing the phenomena, by definition of a pattern model-and equations of the "mechanistic model" that are explaining the phenomena, and regarding which one should ask whether the mathematical

\footnotetext{
4 The homonymy is damageable but it is present in the literature, therefore I keep the same word, and prefer not to use artificial typographical tools (indices etc.) to indicate the difference; it should be clear enough according to the contexts.

5 In what follows, "mechanistic model" refers to the scientists' common use of distinguishing mechanistic and phenomenological models; "mechanistic explanations" refer to the explanations in which mathematics have a merely representational use, which is taken by the "new mechanicists" as a property of any explanation in neuroscience (at least until examples of the contrary are given).

6 "Mathematical description, while not essential to all mechanistic explanations, is certainly a useful tool for characterizing the complex interactions among components in even moderately complicated mechanisms" (p. 606).
} 
properties - especially topological properties in the present case-are explanatory or representational.

Thus showing that a set of dynamical equations concerns the former and therefore is not explanatory does not say anything regarding the status of mathematics in the latter. Kaplan and Craver (2011) are surely right regarding the fact that dynamical systems modeling patterns are not explanations (stricto sensu, i.e. in the sense of "mechanistic models", because pattern models are only explanatory in a weak, predictivist sense, which is admittedly full of problems), and so that dynamicists like Van Gelder did not debunk mechanicism in neuroscience, but one can't infer that any scientific explanation should obey a mechanicist requisite. What is advocated by the present paper and the ones I cited in introduction regarding structural explanations is that, may be only outside neuroscience, there are explanations (sensu "mechanistic models") in which mathematics have a crucial explanatory role.

\subsection{The kinds of continuum view and their defenses}

Yet confusing "mechanistic models" and the mechanistic claim of "new mechanism" is not the only available objection for those who still deny the view that there are topological explanations. Independently of the basic pattern/process distinction, they could elaborate a continuum view of explanation: one goes along a gradual scale from the most concrete mechanisms to the topological explanations, which are very abstract mechanisms. For instance, the ecology of rabbit hunting by foxes unravels a mechanism with many dimensions: physiological, neurological, behavioural, etc; the Lotka-Volterra equations describing the prey-predator cycles addresses the same system and phenomena, but by capturing a much more abstract mechanism; and topological explanations, that would for example focus on attractors in a phase space defined by the Lotka-Volterra equations, would be even more abstract.

An argument for that view consists in saying that in any case "mechanisms" in explanations already are abstractions: one abstracts away from many irrelevant details, in order to focus on the relevant "activities", leaving out many particularities of each of their instantiations. The mechanism of combustion in classical car engines abstracts away from differences in substrates and position of pieces that are proper to each model of car. Or, the mechanism of light transduction in the retina involved in human trichromatic vision concerns several proteins including opsins, rods, then nervous fibers, etc. The precise genetic sequences supporting these entities will differ across individuals, and sometimes the opsin proteins that capture the photon going through the retina in my eye differs according to its amino acid sequence from another protein doing the same job in someone else, however it is one and the same mechanism that accounts for proton capture and then light transduction (Yokoyama 2000). So why not go on and consider mechanisms defined through an increasing scale of abstractness, the highest ones being the topological ones? According to this "continuum view", the mechanism of lock opening abstracts away from the differences between an old key of the wooden door of a barn and a recent key of the lock of an iron modern door; yet they are the same mechanisms of pushing a set of springs through the adjustment with a set of iron teeth, which constitutes the "door opening" mechanism. Granted, 
one would find now in many hotels magnetic keys consisting only of plastic cards, which of course do not push and pull springs within the lock. However one could still view the process of lock opening by a magnetic key as the same mechanism of lock opening by a metal key, consisting of a correspondence between magnetic sequences (and not physical motifs in iron) that eventually moves some springs and metal pieces. This is a mechanism, yet seen in a more abstract way, since one abstracts away from the nature of the entities involved in the adjustment.

However it is questionable whether an extremely abstract mechanism like a topological structure such as a bow-tie or a torus is still a mechanism. One could arguably object here that, with such an extension, the word "mechanism" becomes synonymous of any explanation and therefore brings no additional understanding to our philosophy of explanation. Therefore a more promising argument in favor of such continuum view could consist in emphasizing the fact that besides activities and entities, the "organization" of these activities (Craver and Bechtel 2007) also plays an important role, because a same set of entities with the same activities but differently organized will yield different behaviors. Thus, someone could just say that the more the "organization" plays an explanatory role in a mechanism, the more one moves along this "continuum" towards the structural explanations-and the less it plays this role, the more you move toward "mechanistic explanation". 7

Yet this kind of continuum view may not provide a robust defense for a strong mechanistic thesis. First, saying that in some explanations the "organization" has a strong explanatory role precisely amounts to saying that in some cases geometry is explanatory. After all, if the organization of the entities in a mechanism-say, the setting of the lock and the key-explains why the door can be opened by the key, this relies on geometrical properties - complementarity between concave and convex shapes, etc. So the continuum view implicitly acknowledges that topological or geometrical properties may be explanatory and not only representational. Woodward (2013) also thinks that there are really some distinctive non-mechanistic explanations, that do not meet any of the essential conditions of "paradigmatic" mechanisms.

Second, it seems that labeling this view a "continuum of mechanistic explanations" is purely conventional. If someone labels it "a continuum of structural explanations", meaning that the more some details such as the nature of entities and activities play a role in explanation, the more the structural explanation tends towards a mechanistic pole, then I see no substantial reason to resist this conception. Thus once one defends a continuum view there is something purely conventional in seeing it as a continuum of mechanisms. Many explanations in physics would be purely structural because they just pinpoint something like an organization-think of the solidity of crystals - whereas one would find in many special sciences these more detailed structural explanations that others labeled "mechanisms". I am not arguing for that, but just noticing that this view does not seem illegitimate once someone supports the continuum view, so that an additional argument is needed to discard

\footnotetext{
7 In the former example of the keys, the "organization", in the sense of the link between two states (correspondence/no correspondence) and the motion of the lock, plays a heavy role.
} 
it and embrace the conception that all explanations situated on this continuum are mechanisms.

\subsection{Epistemological critique of the aforementioned continuum view}

Yet the continuum view as such is still lacking epistemological accuracy, for the following reason. Just emphasizing that organization can play more or less a role in a mechanistic explanation appears problematic because it overlooks the way distinct structural explanations can be distinctively explanatory in virtue of which mathematics is playing an explanatory role. Granted, structural explanations in general are such that some "organization" plays an important role, which is attested to by the fact that the nature of the entities and the activities in the mechanism is quite irrelevant-e.g., the same networks can be instantiated by ecosystems or financial systems and yield similar properties (May et al. 2008). However across various structural explanations, the nature of the mathematical properties that play an explanatory role can be very different, as it was obvious when I listed varieties of structural explanations - a distinction that vanishes when one lumps all of them into the catch-all category "organization-centered explanations". In particular, topology is by definition about invariance across continuous transformations; therefore identifying topological properties means identifying in some systems properties that do not vary under some given continuous transformations, as indicated above $(\S 1)$. Hence topological properties specify some modes of invariance, and therefore, they are very likely to explain some aspects of robustness of systems (as argued in Huneman (2010a)), robustness being always defined in terms of the invariance of some system properties through a range of specific perturbations. There is here a principled link between one kind of explanations (the topological ones) and one kind of explanandum (robustness). But when one claims that these explanations are simply mechanistic explanations with a certain importance conferred to the organizational factor, this essential connection between them and the fact that they explain in priority some robustness features-a connection which defines the specific explanatory nature and scope of this kind of organization-is just obfuscated. Thus, the continuum view that includes all structural explanations within the group "mechanisms where the organization has important effects" overlooks an essential distinctive property of these explanations, a property that appears only by focusing on the kind of structure at stake.

I'd like to contrast this continuum view that I just criticized, which is mostly about the logical characterization of explanations, with another kind of continuum mostly centered on scientific practice. While in the former sense mechanisms and topologies are considered two forms of abstract explanations that give more or less importance to the organization and the structure of the phenomenon they explain, in the latter sense mechanisms and topology would be different explanatory types that are more or less integrated in given theoretical structures or explanatory strategies According to this latter continuum view topologies and mechanisms are distinct in principle, but not in practice separated. The last three sections will explore this idea by considering in detail explanatory practices in ecology and evolutionary biology. 


\section{Topological explanations in scientific practice: pervasiveness and modeling specificities}

In physics, actually, a strong argument for structural explanation has been made, which says that mathematical properties explain some phenomena because these are entailed by them together with the fact that the system in which they happen are instantiating mathematical structures that display these properties. Dorato and Felline (2011) convincingly show that quantum entanglement as well as uncertainty principle are respectively explained by non-communatitivity in Hilbert algebra and by limit properties of Fourier transforms-provided that isomorphisms hold between these mathematical structures and the quantum facts about which we have data. ${ }^{8}$ Nevertheless in the special sciences such as biology or social sciences one may resist this view and claim, with the new mechanicists, that mechanisms are the norm of explanations. I will therefore begin this section about scientific practice by indicating that in some areas of evolutionary biology and ecology, especially since two decades, topological explanations became overwhelmingly common. This will allow me to investigate in the next sections the entanglement between topologies and mechanisms, first in theoretical structures, then in specific explanatory strategies.

\subsection{Why the current pervasiveness of topological explanations?}

\subsubsection{Neutrality}

The concept of "neutrality" in evolutionary biology or ecology most generally denotes dynamics where entities at stake have equal fitnesses-like alleles in the "neutralist theory of evolution" elaborated among others by Kimura (1983). The theoretical meanings and uses of "neutrality" have extended beyond this theory, and provide instances of topological explanations, as I'll argue now. One striking occurrence of topological explanations in evolutionary biology comes from recent developments about evolutionary "fitness landscapes" that rely on the notion of neutrality. These landscapes have been originally designed by Sewall Wright to represent in an abstract space the evolution of allele frequencies in a population. When axes are interpreted as frequencies of each allele, a population is represented as a point on a landscape and selection makes populations climb peaks in this landscape (Wright 1931). ${ }^{9}$ As it is well known, a basic problem for evolutionary biology is that populations can in this model get stacked on local fitness peaks. Wright elaborated a theory called "shifting balance theory" to explain that populations may reach global fitness optima. In his model, when a population is on a local fitness peak, drift is what allows it to get down the peak to another peak (instead of climbing and get stacked), and then selection makes it

\footnotetext{
8 More precisely the isomorphism holds between "data model" and "theoretical model". One could argue that this is one kind of relation between pattern models (a form of data model) and mechanistic model but it is left out of this paper.

9 Or, according to another interpretation, evolution of sets of genotypes in an abstract space-in each case one axis is the fitness, either of the population characterized by a specific repartition of alleles, or of the genotype constituted by the alleles.
} 
climb again. Coyne et al. (1997) raised powerful objections against the mathematical consistency of the shifting balance mechanism and against its empirical instantiation. However recently Gavrilets (1999) noticed that in most cases the fitness landscapes are high-dimensional and therefore our ordinary spatial intuitions do not apply. This especially concerns the notions of climbing and going down hill, which according to Gavrilets are out of place. He instead has shown that in these landscapes there exists "neutral networks", as sets made of equal fitness points, which may stand between different "hills", so that a population does not need drift to get on a peak of higher fitness. ${ }^{10}$ In this case, what is in a population mostly explanatory of the existence of globally optimal traits — adaptations, to go quickly — is the existence of such tunnelswhich obviously is a topological fact. ${ }^{11}$ Therefore, what we have here as a solution of the "local fitness traps" in evolutionary theory is a topological explanation, instead of the mechanism envisaged by Wright. All this is mostly indicative, but it is here to suggest - paraphrasing Darwin — that the "paramount explanatory power of neutrality" pervades current evolutionary science, and that such power is cashed out in terms of topological explanations.

\subsubsection{Networks}

One is generally more familiar with biological or social theories that consider networks, which are themselves pervasive in all subfields of the so called "complex systems theory". They are likely to include explanations based on topological properties. Actually, biologists and ecologists often describe networks at any level of biological reality: trophic webs and interaction networks in ecology, gene regulatory networks that support gene expression in molecular biology (Davidson 1986; Davidson et al. 2003), networks of metabolic pathways in the biology of the cell, signaling networks that accompany them (Fernandez and Solé (2005)), etc. Social networks are obviously an unavoidable aspect of social science and our daily social life (e.g. Granovetter 1973). Elsewhere, disease networks (Barabasi et al. 2011) have recently been reconstructed by considering the co-occurrences of a vast amount of genes in the actually known diseases. All these networks gather a huge number of entities, and collecting data about their relations requires of course automated processes grounded on highpower computing techniques. In molecular biology, it has often been remarked that the "post-genomic era" is characterized by our switching from the level of the gene to the genomic network (e.g. Lamm 2014), and, additionally, our realizing that genomic networks work together with other networks like protein networks, etc. (e.g. Fuente 2009). Networks-not only networks physically constituted by synaptic connections, that used to be approximated by neural networks and logical gates-are also pervasive in current neuroscience, where information processing and distribution, resilience, integration and synchronization in the brain are captured in terms of graphs, several

\footnotetext{
10 "Populations can evolve and diverge along bands of highly-fit genotypes without going across the states with a large number of low-fit genotypes (that is without crossing any adaptive valleys)" (Gavrilets 1999, p. 3).

11 "Extended (nearly) neutral networks are important in adaptation for they can be "used" by a population to find areas in genotype space with higher fitness value." (Gavrilets 2003, p. 149).
} 
of which are being investigated in large-scale scientific projects such as Human Brain or Blue Brain, that aim at mapping the brain ${ }^{12}$ (Seung 2009; Sporns 2012).

Notice here that generally networks need not always be actually interacting networks: the edges can be any case of relations relevant for the phenomenon under study (as in the case in network analysis of voxels in neuroscience (Bullmore and Sporns 2009)). In ecology, when a model is called "spatially realist", metapopulations are often modeled as sets of nodes and edges (Hanski 1999): each node is either a possible habitat for a population, or a set of populations in what is called an "island", which can then include several habitats (Huth et al. 2015). There is no genuine interaction between nodes, except in the sense of a virtual migration interaction. Rather there are simply possibilities of a population to migrate or disperse from node to node, colonize other islands with given probabilities, etc.

Granted, networks are a useful way to represent and describe data gathered by sophisticated devices (microarrays, data mining etc.). But what is epistemically proper to this network modelling is that the topological properties found in the networks are such that they explain some of the properties one is interested in, in the way depicted above, namely, the instantiation of these properties is explained by the fact that the network is of such topological nature. The latter indeed yields some lawful regularities, or at least some counterfactual connections, for instance-considering my two examples above: had many species gone extinct in the community, the overall functioning pattern would not have changed; or: should an attack by pathogens happen in the system, CD4+ T-cells would probably be targeted. Here below are examples of such explanatory resources.

In a paper about "topological analyses of cellular networks", Rodriguez Caso and Conde Pueyo noticed: "[a] network, more than recovering a biological process, gives us a conceptual picture" (2009, p. 261). This means that the explanation provided by network analysis is not one concerned with the discovery of mechanisms, but instead conveys a specific kind of understanding of the system under focus. Accordingly, in their paper, the authors model the genes in a cell according to whether being simultaneously deleted is lethal for the cell or not. This allows them to build a graph (Fig. 1) that immediately tells you something about the robustness of the cellular network without needing to understanding its mechanistic functioning, since the amount of genes that are likely to be lethal when deleted with any one of the many other genes in the network is very low. ${ }^{13}$ When the explanandum is such robustness, rather than the functioning of the metabolic network in the cell yielding some specific outcomes, then the topology of this co-lethality network is explanatory, for it entails that many alterations of alleles will only marginally affect the survival of the cell since chances are low that the next alleles to be altered will be exactly the ones connected to them in the co-lethality network.

\footnotetext{
12 "Connectivity analysis has already led to a number of new insights about brain organization. For example, segregated brain regions may be identified by their unique patterns of connectivity, structural and functional connectivity may be compared to elucidate how dynamic interactions arise from the anatomical substrate, and the architecture of large-scale networks connecting sets of brain regions may be analyzed in detail" (Behren and Sporns 2011, p. 144). But see Craver (forth.) for a defence that these models are not explanatory.

13 Notice that the edges in the graph do not represent interactions but relations defined by the result of some interactions.
} 
Fig. 1 Co-lethality network of pairs of genes (after Rodriguez Caso and Conde-Puyeo 2009)

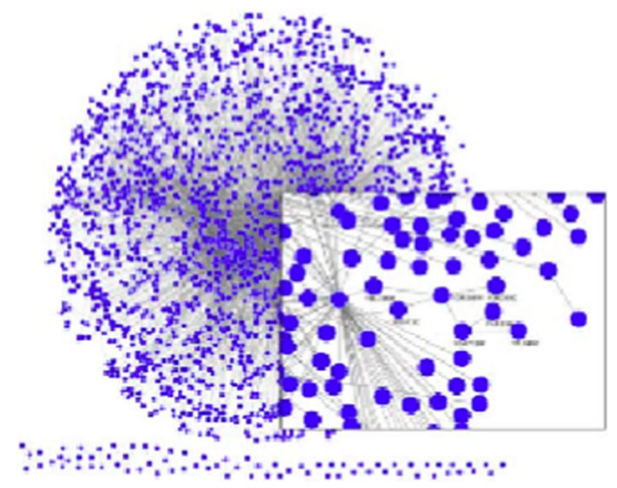

At a more general level, Klemm and Bornholdt (2005) study the reliability of biological networks in abstracto, with respect to stochastic fluctuation in the timing of operations done at each node. They compare various networks and find that the sets of networks contain several networks as attractors. In some of these, fluctuations in timing result in desynchronizing the network so that the global outcome of the network activity is disturbed, while in others synchronicity is kept, hence the global outcome resists fluctuation in operation timing. The latter networks are called "reliable", the former "unreliable". Moreover, those dynamical properties appear to depend upon networks' topologies, characterized by some properties of the 3-nodes subgraphs that compose them. Then one finds that in nature reliable attractors are more frequent than unreliable ones in the architecture of extant networks, which suggests that there has been selection for these topological properties. Reliability regarding timing fluctuations is indeed a plausible selective advantage; e.g., when thermal fluctuations or chemical inputs slow or accelerate the operation of some enzyme, it's advantageous that the set of operations supposed to yield one specific cell activity remains preserved because the timing disturbance will not get echoed and amplified through all other operations. In this study, the topological approach obviously provides us with insights that an inventory and comparison of mechanisms of desynchronizing and resynchronizing could not reach (not to speak of the evolutionary lesson that is then drawn from the empirical data). Identifying reliable networks uncovers a lawful regularity in the way these biological systems are likely to behave in the face of fluctuations in timing, and accounting for such reliability by the topology of subgraphs explains this regularity.

\subsection{Scientific practice: a pragmatic distinction}

Having so characterized the scientific contexts rich in topological explanations allows me to reconsider the question of the distinction between mechanisms and topologies from the viewpoint of the practice of science. It seems that viewing all explanations as mechanistic also overlooks some crucial differences from the viewpoint of explanatory practice, because what scientists do when they elaborate topological explanations is very different from what looking for mechanisms consists in, as I'll explain now. In other words, even if someone resists the arguments given above about the prin- 
cipled distinction between topological and mechanistic explanation, some attention paid to explanatory practice requires making this distinction at least with the purpose of describing what scientists are actually doing.

Regarding network analysis, practitioners often emphasize a principled specificity of their modeling: Rodriguez Caso and Conde-Puyeo (2009) say: "it opens the possibility of a global comprehension of the system, against the predominant reductionism of the current scientific thought" (p. 263); Ulanowicz, a longtime promoter of network perspectives in theoretical ecology, sees graphs as "an alternative to mechanisms" (Ulanowicz 2011, p. 35). His argument is that mechanistic modeling, which looks for functional roles of entities in an organization, often appears problematic and that network thinking is defined in an alternative way: "problems arise, howeverhe writes-as soon as the scope of the modeling exercise encompasses more than one biological process. In most cases, models of multiple interacting processes are less than robust and of marginal utility" (ibid.). He means that when understanding one complex biological or ecological system for which a huge set of data is given, many interacting mechanisms should be modeled but the modelling won't be robust in the sense that another similar system with slightly different data will require important changes in this model: eventually, this modelling cannot capture the common features of systems in which the theory will be interested. Network modelling is an alternative to this quest for mechanisms. So he goes on: "One way to address multiple biological processes is to forego the specification of dynamics and see what can be learned about the system by focusing on the observed configurations of system processes. That is, one effectively truncates the modeling process after the first two steps-identification and parsing. (...) The assumptions that comprise these two steps can be represented as a set of boxes (nodes) that are connected by lines (arcs) denoting the pertinent interactions." (ibid).

This indicates that the scientific practice of making topological explanations (in the form of graph theory) is different from the usual methodology: it is "truncated modeling", meaning that after having identified and parsed the interacting components (modeled as nodes) one just stops the modeling process and does not specify their functional roles or activities. This makes full sense when considering the modalities of current sciences such as genomics, proteomics or community ecology: we gather huge amounts of data, be they from microarrays, websites metadata, scanning of schools of fish by electronic devices, etc.- - and one could not in a finite amount of time capture their specific activities or causal roles. Modeling the pure networks of interactions without searching for mechanisms and functions overcomes this practical intractability. Then Ulanowicz (2011) concludes: "A surprising amount can be learned about how the system is functioning from the topology of the resulting graph or network of interactions." (p. 35) This expresses the intended virtue of such explanations: the topology informs us about some dynamical features_-such as, in my ecology example, the stability of the system. Getting into the mechanisms is not useful, and it can even be detrimental because as soon as one is interested in a class of dynamical processes with some hypothetically common properties, what is really explanatory is the structure of interactions, hence the mathematical properties, that can be variously instantiated by various mechanisms. Thus, the continuum view criticized above obfuscates this 
radical difference in the scientific practice named "truncated modeling", and just for this reason it is useful for the philosopher of science to resist it.

\section{Practical articulation of topologies and mechanistic explanations in theories : constraint and levels}

In what precedes I have shown that topological explanations are a distinct and pervasive mode of explanations in life sciences, and that a philosophy of science attentive to actual practice should in any case make a distinction between them and mechanistic explanations. However, it is true that concrete explanatory practice in general does not use either modes of explanation separately but joins them in concrete model building protocols, explanatory strategies or theoretical constructions. The following sections will explore this articulation between topological and mechanistic explanations. Sections 4 and 5 will consider how theoretical structures of subfields in life sciences or ecology are involving specific articulations between mechanistic and topological explanations. More precisely, Sect. 4 explores usual features according to which in these domains topologies can constrain mechanistic explanations, or both be together articulated within an interlevel theoretical structure. Section 5 will investigate a less studied feature, namely the fact that mechanisms can condition topologies-which is often advocated by tenants of a mechanistic view-but also the other way round, topologies condition mechanisms. These two sections are therefore explicating what it means that topologies and mechanisms are combined in a given theoretical structure or explanatory strategy. Section 6, more exploratory, will show how some recent model-building practices realize an integration of mechanisms within topologies in singular explanatory schemes.

\subsection{Topologies as constraints}

A first kind of articulation between mechanisms and topologies may be termed "constraint". Generally speaking, the topology of a space may more or less constrain the dynamics of a system. For instance, when the connexity is very low in a space, it means that the possible pathways between two points will be much longer-hence, the motions will be slower - than in a more connex space, and hence the trajectories are quite constrained. This relation of constraint extends to the relationship between explanations: topology may often be a constraint on possible mechanisms for a phenomenon, in the sense explicated now.

The same mechanism can yield different outcomes according to the given topology of the system or some space associated with the system. Consider a very general kind of mechanistic explanation, which is provided by reaction-diffusion models (RD). As it is well known, in this model several (two) types of particles diffuse in space, and undergo a specific reaction when they make contact. The fact that the "reaction" is chemical is not essential to the general form of the reaction-diffusion model: what counts is the fact that the dynamics of the system can be described according to a set of equations analogous to those that would describe diffusion and reaction in a system made of two chemical substrates: 


$$
\partial \mathrm{U}(\mathrm{x}, \mathrm{t}) / \partial \mathrm{t}=\mathrm{D} \Delta \mathrm{u}(\mathrm{x}, \mathrm{t})+\mathrm{f}(\mathrm{u}(\mathrm{x}, \mathrm{t}))
$$

where $\mathrm{u}(\mathrm{x}, \mathrm{t})$ is the concentration of a substance in space and time, $\Delta$ is a Laplacian operator, $D$ is a diffusion coefficient (or a matrix of these coefficients), and $f(u)$ is the chemical reaction - the whole equation displaying the addition of a diffusion term and a reaction term. Turing famously applied RD to the emergence of patterns through organismic development (Turing 1952); RD models have also been successful in modeling ecological phenomena, in epidemiology and sociology of collective behaviors, e.g. dynamics of crime hotspots (Short et al. 2010). In epidemiology for instance, "diffusion" is the motion of individuals (infected or healthy) in space and "reaction" is the infection of one person by another according to a given probability: the relevance of RD models here seems therefore straightforward.

"Mechanism of reaction-diffusion" is a concept that does not specifically pinpoint physical entities and that is able to refer to the same thing across several ontological fields; this reading of "mechanism" makes sense of the idea acknowledged above that mechanisms can be quite abstract. Yet, in such cases the topology has been proven to be very constraining regarding the mechanism's outcomes, as investigated by Colizza et al. (2007) regarding RD models in ecology, when considered in the context of metapopulations-i.e. networks of populations. In such a model reactions take place in nodes (populations); "reaction" means ecological interactions or infectious interactions such as in epidemiology. Networks generally speaking can be "homogeneous" or "heterogeneous"- - depending on whether the network properties are distributed across nodes in a very homogeneous and regular manner, or in a very heterogeneous manner, such as heavy-tailed or scale-free networks. If the network is homogeneous, Colizza et al. found that the behavior will be very different than what happens in heterogeneous networks; importantly, the occurring dynamics may not exhibit a phase transition at the thermodynamic limit. Network topology clearly constrains the outcomes of RD.

This parallels a very general finding in graph theory: heterogeneous networks, thanks to their architecture, possess specific dynamical properties regarding stability, robustness, resilience etc., as it is exemplified by the case of scale-free networks cited above (Strogatz 2001). Recall that in heterogeneous networks nodes and edges are not homogeneously distributed; thereby, to put it bluntly random networks are likely to be homogeneous. Hence the more you depart from random structures the more topological properties appear explanatory of interesting phenomena.

The constraining relation comes in a matter of degrees. At the limit, the topology can be so constraining that the dynamics of the system will be fully determined, as I will illustrate by the behavior of networks regarding robustness. In a random network nodes are randomly connected, with an average degree K. Let's consider two-states models where a node is active or inactive and influences its connected nodes (i.e., switches it on or off) with velocity r, or, more abstractly, through an interaction that takes place at a rate $r$. If the connectivity of the network goes from randomness to scale-freeness (where the nodes are connected in a very heterogeneous way), the system's robustness will increase, as shown by Aldana and Cluzel (2003). In their model they consider the coefficient of scale-freeness called "scale-free exponent" $\gamma$, which is the $\gamma$ power in the equation describing the probability distribution of nodes as the probability that an 
arbitrary node is connected to k nodes-which is given by: $\mathrm{P}(\mathrm{k})=[\mathrm{Z}(\gamma) \mathrm{k} \gamma]^{-1}(\mathrm{Z}$ being a normalization factor).

They found that when $\gamma$ is low $(<2)$, then some networks will be robust enough to withstand perturbations of the connections, whereas others will be chaotic, depending upon the value of $\mathrm{r}$ and $\mathrm{K}$. However, beyond a threshold above 2.5, that is, when the scale-free exponent is high enough, almost all networks will be robust, meaning that the connectivity $\mathrm{K}$ or the rate $\mathrm{r}$ are not affecting robustness any more. Robustness of the dynamics is very generic and does not depend upon $\mathrm{r}$, which describes a property of the mechanism at stake. At this point, the topology wholly constrains the dynamics (at least regarding robustness properties).

\subsection{Interlevel combination}

It has been convincingly argued by many philosophers of science interested in complexity that most systems are made up of several levels of subsystems (e.g. Wimsatt 1972). Furthermore, frequent explanations that in general consider one level of a subsystem differ in kind from explanations that consider the interlevel relations. Put in terms of mechanisms, at one level there are entities, which interact with one another to produce a specific output, while each entity can itself be considered a subsystem characterized by its specific mechanism. In this context Craver and Bechtel (2007) emphasized that interlevel explanation and intra-level explanation differ: whereas causation is proper to the relationship at a specific level, interlevel relations are simply constitutive and not causal. ${ }^{14}$

Generally speaking, suppose we have a system $\mathrm{S}$, and subsystems of $\mathrm{S}$, calling them $\mathrm{M}_{\mathrm{i}}$; suppose we have also $\mathrm{N}_{\mathrm{j}}$, which are the parts of subsystems of $\mathrm{M}_{\mathrm{i}}$, which, taken together as a mechanism, cause $\mathrm{M}_{\mathrm{i}}$ 's outcome. They don't cause S's activity, rather they contribute constitute it. Yet, even if this distinction seems interesting, it is nonetheless unnecessary that the intra-level explanation (focusing on the $\mathrm{N}_{\mathrm{j}}$ ) should be mechanistic. Instead, one could imagine, across various levels, an alternation of mechanisms and topologies. Rather than outcomes of mechanisms at level $n-1$ the mechanisms at level $n$ could be constituted by topological structures yielding a regular output at this level.

Here is an imaginary example. Suppose a version of the Gaia hypothesis in which Earth is a complex adaptive system endowed with some physiological feedbacks that support its stability (relative constancy of properties such as biomass, productivity) and resilience as well as its inhabitability. ${ }^{15}$ One can think that the functioning and stability of this Earth, $\mathrm{S}$, is constituted by several ecosystems $\mathrm{M}_{\mathrm{i}}$, whose stability contributes to the Earth's overall stability. They are entities, and their combined activities therefore

\footnotetext{
${ }^{14}$ For example, Craver (2007) writes: "there is a temptation to say that the activation of cyclic GMP phosphodiesterase, which catalyzes the conversion of cyclic GMP to 5c/-GMP, causes rod cells to hyperpolarize, which in turn causes the eye to transduce light into neural activity. But the activation of cyclic GMP phosphodiesterase is part of the activity of depolarization, which is part of the eye's transduction of light" (p. 15).

15 This paper is not committed to the validity of any version of the Gaia hypothesis, the example is just chosen for its simplicity.
} 
constitute the overall behavior of the Earth. In turn, one can investigate the constitutive subsystems - the singular ecosystems - as mechanisms producing stability through internal feedbacks between their elements, the species $\mathrm{N}_{\mathrm{j}}$. This corresponds to the above schema of a system $\mathrm{S}$ constituted by subsystems $\mathrm{M}_{\mathrm{i}}$ within which entities $\mathrm{N}_{\mathrm{j}}$ mechanistically cause the subsystems' outcome and therefore constitute the overall system's behavior and outcomes. However, the robustness of ecosystems can also be explained, as we have seen, through topological explanations; in this case, the constitutive relation that holds between levels would tie topological explanations at the level of each $\mathrm{M}_{\mathrm{i}}$ - the ecosystems - and mechanisms that consider each $\mathrm{M}_{\mathrm{i}}$ as an entity playing its role at the level of the overall mechanism of the system of Earth. This toy example was intended therefore to show that topological explanations and mechanistic explanations can be combined into an interlevel theoretical approach.

\section{How types of explanation condition each other in a given theoretical structure: with an emphasis on Modern Synthesis evolutionary theory}

\subsection{The most usual understanding of the "condition" relation}

Another important way to understand the articulation between mechanisms and topological explanations in extant theoretical structures consists in viewing how one conditions the other. This is first obvious when one considers the type of question each is supposed to answer, as made clear by the following example.

(i) In the case of ecological stability, topological explanations (in terms of scale-free networks) answer the following question: "why is such a community, one that features this specific web of interaction, stable?"

(ii) However, once this explanation is given one could also ask: "why is it that this community has a scale-free network?" This question will receive an answer in terms of a mechanistic explanation. As it has been shown by Albert and Barabasi (2002), the mechanism of "preferential attachment", in which the probability of interacting with another entity increases in proportion to the number of interactions the entity already, causes scale-free networks when it is used as a rule to establish edges when building a network, and is indeed their most common cause. Hence the answer to our question (ii) will probably be an ecological interaction mechanism that realizes preferential attachment.

This epistemological schema may also contain another way topologies and mechanisms connect, namely, when random topology provides null models to assess mechanistic explanations. If a random network yields the properties of the system in which we are interested, it may not be necessary to look for additional specific mechanisms that would account for such properties. A simple example is provided by the network of CEOs in America. Here, considering the number of boards where two CEOs sit together, one can reconstruct the network of CEOs (see Strogatz 2001). The clustering coefficient $\mathrm{c}$ of the network captures the probability that two CEOs who share a board with another one share a board together. Actually, a random network yields a value of c close to the real data. In contrast the network of cowriting scientists is more clustered than what is expected randomly. The conclusion is that 
no specific social force should be hypothesised to explain the network of CEOs since when it is randomly constituted, unlike in the case of scientists, the graph predicts the correct coefficient of the CEOs' network. Hence the random network constitutes the null hypothesis for explanations in terms of social mechanisms.

Something similar affects current evolutionary biology. In analyzing Gene Regulatory Network, Uri Alon two decades ago has shown that many small configurations called "motifs" (for instance made of two interaction triangles between regulatory genes) are pervasive across different clades and phylogenetically conserved (see e.g. Alon 2007). Such pervasiveness yields the hypothesis that there has been long lasting selection for these motifs. However Solé and Valverde (2006) argued that many of these "motifs" are in fact easily obtained from the mere construction of graphs by random node duplication (gene duplication being a realistic process in evolution), and therefore, that optimality arguments inferred from the mere pervasiveness are here not supported since a null hypothesis is equally predictive. So we see that not only specific networks indicate plausible mechanisms, but random networks of many kinds play also the role of a null hypothesis for hypothesised mechanisms.

(iii) A third kind of question one can ask is: "why is it the case that most of the communities known in ecology have a truncated scale-free network?" An answer to this question may indeed appeal to general features of the ecological mechanisms of interaction.

This last sort of question, however, may not in principle require a mechanistic answer. In some cases, for example, the fact that scale-free networks are pervasive may call not only for a mechanistic explanation in terms of mechanisms that provide preferential attachment, but also an optimisation explanation that relies on natural selection, since the pervasiveness here indicates some optimality property (Aldana and Cluzel 2003). One could then make a case that optimality explanations are structural explanations (given their relative indifference to the mechanisms supporting the optimisation, as indicated in the introduction above; see also Rice 2012). However, the inference from pervasiveness to optimality is not in general legitimate, and one would require a null hypothesis to compare with it, as we just saw; anyway no major views in the present paper rest on such a claim, whose elucidation is left for another paper.

Nevertheless, while it's almost trivial that topologies may be conditioned by mechanisms, the conditioning relation may also obtain in the other direction, which is more interesting. In effect, in many cases the mere validity of a mechanistic explanation, in the sense of the assumptions that are made in order for this explanation to be valid regarding the explanandum, pertains to topological facts - which allows one to talk in terms of "conditions of possibility". To show this I will consider the case of standard population genetics.

\subsection{Conditioning relation in evolutionary biology: topologies as condition for some mechanistic explanations}

Classical evolutionary biology is known as the "Modern Synthesis". It originated from the synthesis of Darwinian natural selection and Mendelian genetics through the works 
of the first population geneticists (Fisher, Sewall Wright, Haldane, Huxley), and then expanded to other disciplines such as systematics and paleontology (e.g. Mayr and Provine 1980, Gayon 1998). According to Modern Synthesis, and notwithstanding the major conceptual and methodological disagreements that existed between all its architects, as Huxley put it in a letter to Mayr in 1951 "natural selection, acting on the heritable variation provided by the mutations and recombination of a Mendelian genetic constitution, is the main agency of biological evolution". For this reason, population genetics, as the science of the change in frequency of alleles under the action of natural selection, is crucial. Actually, notwithstanding their major disagreements on many philosophical, methodological and biological issues, Modern Synthesis biologists conferred to population genetics a central role in evolutionary science, since it captures the process of evolution by natural selection. As Lynch (2007) provocatively puts it: "Nothing in evolutionary biology makes sense except in the light of population genetics."

For the sake of this paper, I will consider the explanations in population genetics as mechanistic explanations: the entities are the alleles, which are associated with genotypes, they recombine, they mutate, and they replicate with a given probability defined by the fitness value of the genotypes they are in at their locus, which averages across the individual organisms sharing such genotypes and becomes what is called "trait fitness" (Orr 2009). The very question of whether this is actually a mechanism in the sense of "new mechanicism" is open (see Skipper and Millstein (2005) for a critique), but even if it's ultimately not the case-which I'm rather ready to admitstill, population genetics is undoubtedly a specific dynamics of alleles (Grafen 2007; Huneman 2014), exactly in the sense Newtonian science is a dynamics of motion of masses - and the question I'm addressing now could then be rephrased in terms of the relation between dynamical explanations (sensu classical dynamics) and topologies. Even if strictly speaking the mechanicist view does not apply to population genetics, it is still legitimate to see it as analogous to Newtonian mechanics. This analogy is indeed pervasive in the language of population genetics, as is clear when reading the founders of the field.

The main point that I want to develop below is that the legitimacy of population genetics as a mechanistic (in a broad sense) explanation of evolution hangs upon several assumptions that concern topological facts. These facts can be distributed into three clauses, given here in an indicative way-they might be not fully independent, but a detailed specification is left for future work.

\subsubsection{Non-ruggedness clause}

Kauffmann (1993) initiated a new way to question the ability of natural selection to really promote new adaptations. Defining types of genotypes by the relation between the number of genes $\mathrm{N}$ and the number of functional links (epistasis) $\mathrm{k}$, he modeled genotypes as Boolean networks, and considered the space of all these networks and their fitness values - which is a fitness landscape. He found this famous result that, to be likely to support evolution by natural selection in the way population genetics characterizes it, these genotypic networks should have a particular nature- the fitness landscape they yield should be not too rugged, where ruggedness is a direct 


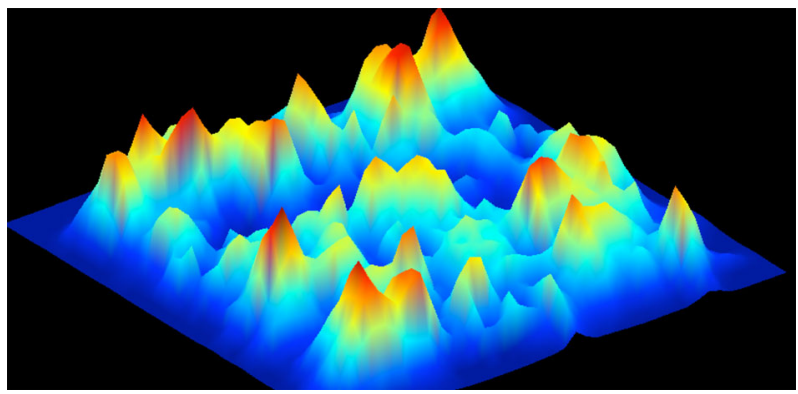

Fig. 2 Rugged fitness landscape of Nk genotypes (Wikipedia commons)

function of $\mathrm{k}$ and $\mathrm{N}$ (Fig. 2): the ruggedness of the landscape can be weakened simply by decreasing the parameter $\mathrm{k}$. If landscape is too rugged, then selection will not be able to target high fitness alleles, retain them and associate them within high fitness genotypes likely to support high fitness traits, and hence natural selection is just not the mechanism of adaptive evolution. In Kauffman's view, this leaves room for other mechanisms such as selforganisation; and this also opens the question of the evolution of these Nk networks. Clearly here the validity of a specific mechanism for adaptive evolution depends upon a topological condition (non ruggedness). On the basis of this well-known work, I will point out two additional conditions for using the mechanisms of population genetics as explanation of extant adaptive evolution.

\subsubsection{Second condition: landscape topological simplicity clause}

We could generalize Kauffmann's approach and thereby, besides non-ruggedness, sketch a second condition for population genetics mechanisms as explanatory of adaptive evolution. Leaving aside issues about the adequacy of fitness landscapes to high-dimensional realities mentioned in the first section, I will just highlight one of their general features, independently of Kauffmann's modelling. If the landscape models the fitness of various genotypes, one has to be conscious that in reality this landscape is itself evolving - namely, the fitness of one genotype is often a function of the frequency of this genotype and the others in the population (frequency-dependent selection) - but this frequency precisely changes under the effect of natural selection and other evolutionary forces, which in turn modifies the landscape. To this extent, Lewontin (1974) used to compare fitness landscapes to a carpet relying on water: the shape of the carpet evolves when someone walks on it.

Taking this into account, it appears that systems in which the mechanisms of population genetics yield adaptive evolution obey an important topological constraint. Suppose indeed that the shape of the landscape changes drastically after each generation. In this case, neither natural selection nor drift - that are by definition responsible of these changes - could lead the population to a new equilibrium. The mechanisms of population genetics exist here but they can't be appealed to in order to explain interesting phenomena regarding equilibria or non-chaotic frequency change in the 
population. ${ }^{16}$ In order to make such mechanisms into actual adaptive evolution mechanisms the relevant assumption about the landscape would indeed be the following: between two not too distant steps, like $\mathrm{t}$ and $\mathrm{t}+2$, a given neighborhood in the landscape should not be too distorted, in the sense that two arbitrary points in it should on average not be too remote from one another. Otherwise the drastic change of landscape evoked above is always possible, and the selection acting on traits in a classical manner will be ineffective. Of course the clause so stated is of topological nature (invariance of neighborhoods through deformations), thereby the explanatory force (regarding adaptive evolution) of the mechanism called "dynamics of gene frequencies" is topologically conditioned. ${ }^{17}$

\subsubsection{Third condition: the developmental clause}

Another condition for viewing the mechanisms of population genetics as explanantia of evolution and especially adaptive evolution concerns the ongoing debates regading the role of development in evolution. Critiques of the Modern Synthesis, especially those from evolutionary developmental biology, often claim that this view unduly dropped development out of evolution: from the viewpoint of population genetics evolution is only an affair of gene pools, and the pathways in a given environment through which genotypes give rise to adult phenotypes that reproduce are irrelevant to evolution (Raff 1996; Gilbert et al. 1996; Amundson 2005). Indeed through its simplifications population genetics classically only takes into account genotypes, traits and their reproductive chances, not development-thus, accepting the anti-Modern Synthesis critiques would therefore lead to pushing population genetics away from its central role in evolutionary biology as the science of the process of evolution. One way to settle this controversy consists in considering the space of possible phenotypes, the space of possible developments, and the space of possible genotypes-as well as the applications that map each one onto another (Huneman 2010b). Thus, the question of the relevance of development to evolution becomes the following: which kind of application between these three spaces is the actual one (at least for a given problem, for example, the microevolution of the Drosophila population, the macroevolution of the clade of salamanders, etc.)? Actually, these kinds of applications can be characterized topologically, according to whether or not they conserve the neighborhood around one given genotype, when the genotype space is injected into the developmental space and then into the phenotypic space (Fig. 3) - in the latter case, neglecting what happens in the developmental space would clearly lose important informations concerning the evolutionary process. Applications between these spaces can therefore be divided into two types: the ones that belong to the less conservative type, and therefore do not

\footnotetext{
16 I'm of course not claiming that frequency-dependence prevent natural selection to lead to equilibria, since such equilibria are pervasive in behavioural ecology. The point is rather that frequency-dependent selection models, when they account for adaptive evolution and extant equilibria, do implicitly assume this clause, which is indeed rather mild.

17 Note that the status of this clause parallels the condition of heritability, necessary for having evolution by natural selection - if heritability is too low, natural selection may change frequencies of traits and alleles but only for one generation, and thus no evolution (and especially the cumulative selection that gives rise to adaptive evolution) would be possible (Brandon 2008).
} 

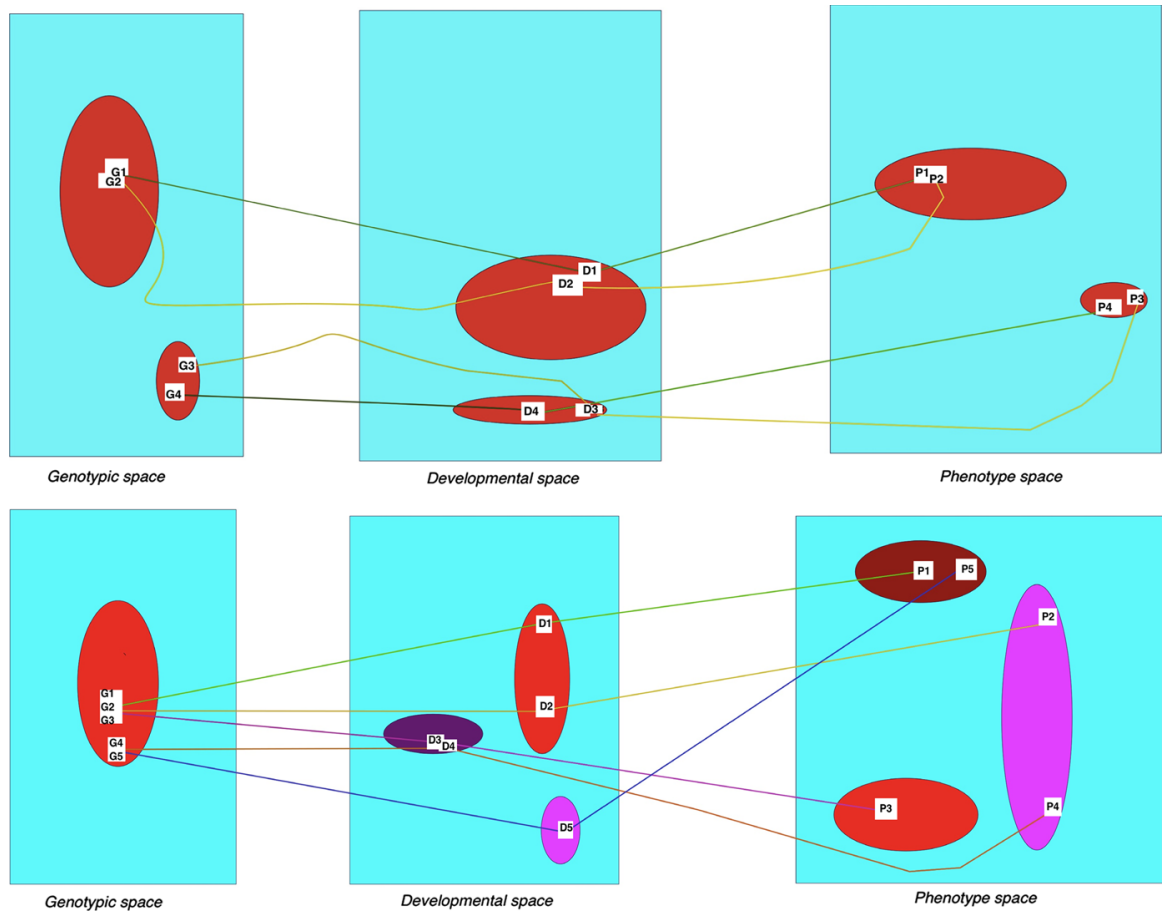

Fig. 3 Two types of applications between Genotypic, Developmental and Phenotypic spaces (after Huneman 2010b). In both figures I use a same colour to draw the projection of a Genotype Gi onto a specific development $\mathrm{Di}$, and the projection of Di onto a Genotype Gi. The ellipse represents the neighbourhood of some given points $\mathrm{Gi}$, Di or Pi in each of the spaces. In type 1, above, when two genotypes G1 and G2 (or G3 and G4) are very close, their images in developmental space, D1 and D2 (resp. D3 and D4) are also in the same neighbourhood, as well as the images of these P1 and P2 (resp. P3 and P4) in the phenotypic space. In type 2, below, when genotypes, e.g. G1...G5, are in the same neighbourhood, the application that links them to the developmental space is such that a given neighbourhood (e.g. G1..G5) is not mapped onto a single neighbourhood, and moreover, the images D1...D5 of G1...G5 in the phenotypic space are also distributed in distinct neighbourhood so that the topological structure of the developmental space is not either conserved. In this case, because the image-image of points in the genotypic and the developmental spaces are scattered, I used different colours to represent them

respect what we could call a clause of topological simplicity, are not likely to allow us to bracket development as population genetics' simplifications intend, and therefore are the ones in which the explanatory power of population genetics (construed in terms of the dynamics of alleles) is lessened. Hence the validity of population genetics as the accurate model for evolution is conditioned by a topological condition regarding these triplets of spaces.

\subsubsection{Summary}

Hence, the developmental clause (4.2.3), the landscape simplicity clause (4.2.2), and the non-ruggedness clause (4.2.1) (which may be a specification of the landscape clause, and is not itself unrelated to the developmental clause and its concern about 
the applications between spaces) are three (possibly overlapping) sets of topological facts that condition the relevance of mechanisms of population genetics to explaining evolution.

This short excursus into evolutionary biology aimed to show that in reality, the conditioning of topologies by the mechanisms that build topologies is not the only kind of conditioning relation between mechanisms and topologies, but that the reverse relation also obtains. Once we have considered how topologies and mechanisms differ, and how in principle each of these explanations can be related to the other within the explanatory structure of a science, I turn to another question of about their relationship: to what extent can a given model be in itself an integration of mechanistic explanation within topological explanation? This is another way to make sense of the type of continuum view defended here-namely, that these two explanatory types are not absolutely opposed to one another, being distinct in principle (Sect. 2) but not in practice separated.

\section{Integrating various kinds of explanations in one model-building strategy}

Besides the articulations of mechanisms and topologies in theoretical structures, as explored below, one witnesses that in practice particular models often integrate to some degree mechanistic informations into topology. Most generally, besides rough graphs that I mostly considered until now, many networks are represented by weighted graphs, i.e. a value of interaction is affected to each edge. This arguably imports an element of mechanistic description into the mere topology, contrasting with rough graphs in which topologies seem explanatory per se. But the integration of mechanism within topological modelling can take various forms, instantiated by several advances in network modelling that I will examine in this section.

\subsection{Merging ecological networks}

An advance in network approaches to ecological systems addresses the merging of networks of various natures. This paper ends with an analysis of such a project because it allows us to raise a crucial issue regarding the relation between topologies and dynamics, namely, the question of timescales.

First the context should be described. Network approaches in ecology have been developed for three decades (Pimm 1985 being a milestone). These approaches allow researchers some insight into the reasons for various kinds of stability, robustness, or contrarily, fragility of ecological communities (Justus 2008, on stability). Many initial networks were food webs, i.e. trophic webs, where the edges instantiated a relationship of predation between two species. However, some networks have been defined by considering all ecological interactions on a par, each instantiating a particular edge (Solé and Montoya 2001). These very general networks, which at best illustrate the detachment of the nature of mechanistic interactions proper to topological explanations, are still less elaborate than food webs, which are by far the best known kind of ecological network, the study of which was pioneered by Elton (1927) (Fig. 4). 


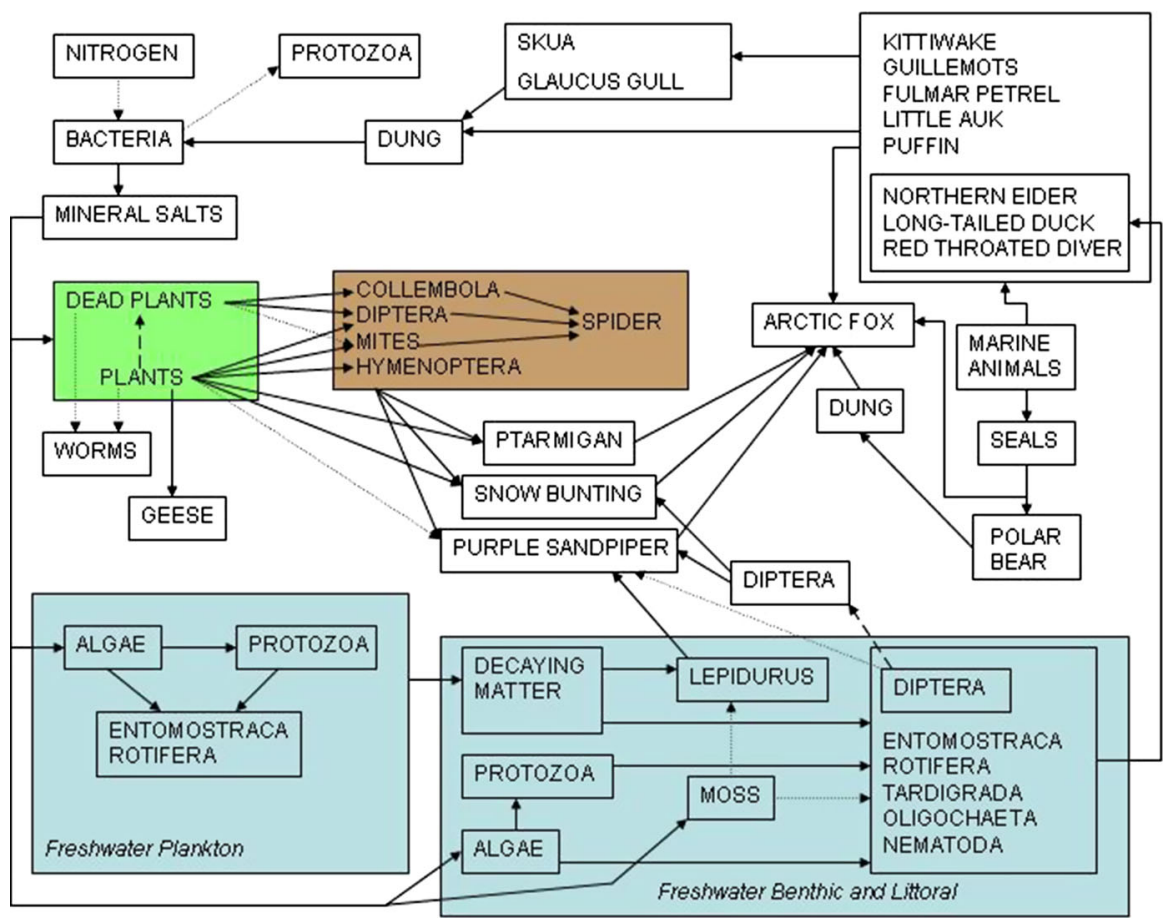

Fig. 4 Elton's representation of food-cycle on bear island, Spitzberg (Elton 1927)

But focusing on trophic networks entails the implicit assumption that predation is the most important ecological interaction to track for understanding community stability or ecosystem functioning. Notwithstanding the important results of food web studies, and especially the impact they had on conservation biology, recent research gave up such an assumption. As Olff et al. (2009) write: "studies that use food web theory to better understand a particular ecosystem thus implicitly assume that predation is the most important process that regulates the abundance of organisms in that ecosystem. Inclusion of non-trophic interactions broadens food web studies to the analysis of interaction webs" (p. 1756, my emphasis). Therefore the task consists in integrating all kinds of interactions within ecological networks - a project in which explanations will be of overt topological nature. As compared to general interaction networks cited at the start, which consider all interactions on a same footing, these studies aim first at considering various peculiarities of the structure of each interaction network, and in a second step, merging the network. The merging itself is of high explanatory value, as indicated in the present example.

Following several focalized studies, Fontaine et al. (2011) classified interactions along two parameters, their level of intimacy (parasitism is more intimate than competition), and whether they are antagonistic (like competition) or mutualistic (like pollinisation). It appeared that the way a kind of interaction behaves according to these two parameters implies that the network of these interactions will be quite specific in terms of nestedness or modularity. Modularity is defined by a high clustering 
coefficient; modules are easily identified when visualizing the network, as relatively separate compartments, and these compartments are characterized by the fact that entities (nodes) within them are more interacting between themselves than with entities in other modules. In contrast, nested networks are such that compartments of this nature cannot be identified; rather, entities tend to distribute their interactions in a homogeneous way across the rest of the network.

The investigation finds that high intimate interactions yield more modular networks whereas low intimate interactions yield more nested networks. "From low to high intimacy, network architecture changes from highly connected and weakly modular to weakly connected and highly modular. Although empirical evidence remains scarce, these results strongly support the conclusion that high interaction intimacy leads to compartmentalization in both mutualistic and antagonistic networks." (Fontaine et al. 2011, p. 1173) On the other hand nested architecture (which minimizes modularity) is more prevalent in mutualistic than antagonistic subnetworks (Fig. 5).

\subsection{Consequences on multiscale modelling}

An important consequence of this finding is that the global ecological network, which is constituted by integrating networks of various interactions, is much affected by these differences in topology of subnetworks. "It appears that merging networks creates important new pathways for direct and indirect interactions". This entails that when considering one subnetwork, for example the plant-pollinisators network in a plant community, the mere architecture of the network does not by itself account for the dynamics even if it is very constraining, because it undergoes the effects from other networks with which it is merged in reality. The investigation of the coupling of networks in ecology therefore bears this important consequence that the behavior of communities regarding perturbations can be better understood, as indicated with the following conclusion: "when two mutualistic networks are linked to each other, a perturbation might be amplified during its propagation through the network because of the presence of positive feedbacks. On the contrary, when the two antagonistic networks are linked to each other, the perturbation could be dampened by negative feedbacks as it propagates through the web. Interestingly, when a mutualistic network is associated with an antagonistic one, the antagonistic network may act as a stabilizing entity dampening the perturbation, while this same perturbation would have been amplified when only the mutualistic sub-network would have been considered." (Fontaine et al. 2011, p. 1177)

Thereby, according to this latter discovery we see that this kind of study directly opens new insights into the evolution of communities, and ultimately, into the connection between evolution and (community) ecology, an issue that has been hotly discussed in the recent years (e.g. Post and Palkovacs 2009; Matthews 2011). It is clear that evolution, as microevolution according to Modern Synthesis-change in gene frequencies in a population in a relatively stable species environment, - and ecology — change in species communities disregarding the population level change in gene frequencies in the given species - often occur on distinct timescales, even though each affects the other. This decoupling allows researchers to model each of them sepa- 


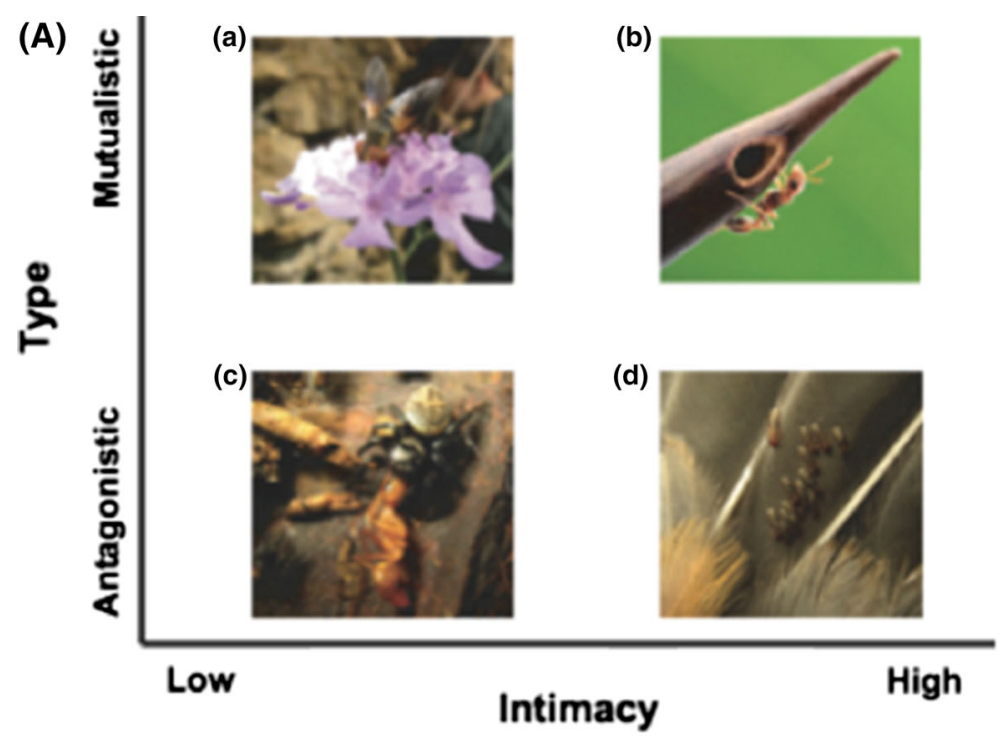

(B)
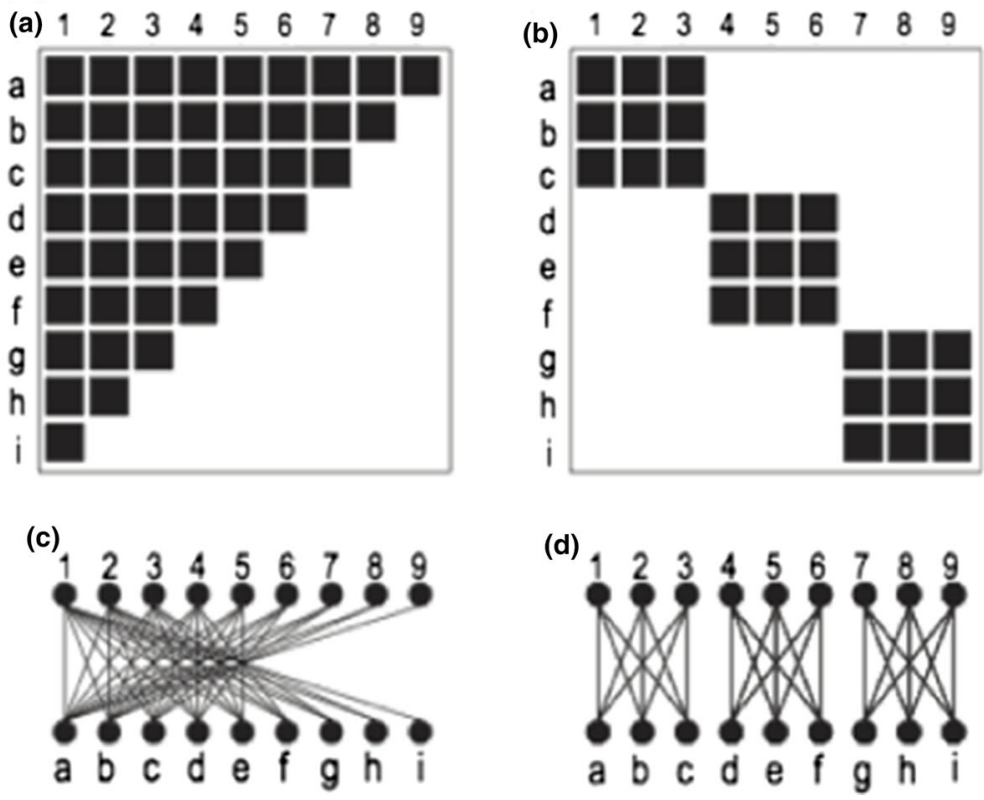

Fig. 5 Typical interaction network of mutualistic networks (right) and antagonistic networks (left) (after Fontaine et al. 2011)

rately. However, we see that the sophistication of topological explanations in ecology and the way one can integrate some differences in processes into the establishing of network architectures and then the merging of networks leads to a better understanding of the way ecology may condition evolution by constraining the fate of the species 
in the networks considered. Therefore, a common approach to evolution and ecology can emerge that overcomes the assumption of decoupled timescales.

This last case was discussed at length because it provides a good example of the integration, within one model, of topologies-i.e., network architecture-and mechanistic explanations-i.e., distinction of subnetworks defined by two interaction parameters. It also shows that in turn evolutionary dynamics can be better understood through the constraints that these topologies impose on the dynamics of the species, in a way that is explained by topological considerations. Now, recall that the timescale of ecology is at the same time longer (since microevolutionary population genetics assume some constancy of the many species environing the focal population) and shorter (since macroevolution occurs slower than change in biodiversity patterns in an ecosystem) than evolution as such. It therefore follows that such study of merging networks, to the extent that it sheds light on the influence of ecology onto evolution, is significant regarding the way an integration of mechanistic explanation and topologies can ultimately provide insights about the coupling and uncoupling of processes at different timescales.

\section{Conclusion}

Topological explanations belong to the general family of structural explanations, which are somehow detached from the consideration of mechanisms proper to the systems under study. Topological explanations are overwhelming in biology and ecology, especially when it comes to understanding stability and robustness properties. I have argued that there are reasons, proper to a philosophy of science interested in the fine-grained practice of science, to resist a continuum view that sees them as an abstract kind of mechanism. I have shown that these two types of explanation have several kinds of articulation in scientific practice: topologies may constrain mechanistic explanations, for instance in the way a network topology constrains more or less the dynamics of what takes place in the network; but more interestingly topologies and mechanisms are likely to condition the explanatory power of each other. A promising research project, sketched above as an example of these conditioning relations, would be the detailed unraveling of the conditions (spelled out in topological terms) for the validity of the assumptions of population genetics as a central explanation of adaptive evolution.

But more recently a given topological modelling strategy may give rise in itself to considerations that integrate mechanistic information. Yet far from making these explanations an avatar of mechanistic explanations, it seems that they display the power of topological explanations to provide deeper insights into real systems or processes by allowing a renewed understanding of the connection between topology and dynamics, as instantiated by the connection between ecological networks and evolutionary dynamics in recent functional ecology.

Therefore it seems that the distinction between mechanistic and structural explanation, and, among these, an emphasis on topological explanations, provides us with interesting tools to make sense of the richness and plurality of explanatory practices, at least in contemporary evolutionary biology and ecology. 
Acknowledgments The author warmly thanks Isabelle Drouet, Daniel Kostic, Carl Craver, Anya Plutynski, Denis Walsh, and the participants of the Topology and mechanisms conference in Belgrade (2013) for their feedbacks (2012). Many thanks to two anonymous reviewers for their hugely constructive criticisms. This work is supported by the ANR Grant Explabio (\#ANR 13-BSH3-0007).

\section{References}

Albert, R., \& Barabasi, A. L. (2002). Statistical mechanics of complex networks. Review Modern Physics, 74, 47-97.

Aldana, M., \& Cluzel, P. (2003). A natural class of robust networks. PNAS, 100(15), 8710-8714.

Alon, U. (2007). Network motifs: Theory and experimental approaches. Nature Reviews Genetics, 8, 450461.

Amundson, R. (2005). The changing role of the embryo in evolutionary thought. Cambridge: Cambridge University Press.

Baker, A. (2009). Mathematical explanation in science. British Journal for the Philosophy of Science, 60, 611-633.

Barabasi, A. L. (2011). Network medicine: A network-based approach to human disease. Nature Reviews Genetics, 12, 56-68.

Batterman, R., \& Rice, C. (2014). Minimal model explanations. Philosophy of Science, 81(3), 349-376.

Bechtel, W., \& Abrahamsen, A. (2005). Explanation: A mechanistic alternative. Studies in History and Philosophy of the Biological and Biomedical Sciences, 36, 421-441.

Brandon, R. (2008). Natural selection. In E. N. Zalta (Ed.), The Stanford encyclopedia of philosophy (Spring 2014 ed.), http://plato.stanford.edu/archives/spr2014/entries/natural-selection/.

Behren, T. E. J., \& Sporns, O. (2011). Human connectomics. Current Opinion in Neurobiology, 22(1), 144-153.

Bullmore, E., \& Sporns, O. (2009). Complex brain networks: Graph theoretical analysis of structural and functional systems. Nature Reviews Neuroscience, 10, 186-198.

Canguilhem, G. (1965). La Connaissance de la vie. Paris: Vrin.

Colizza, V., Pastor-Satorras, R., \& Vespignani, A. (2007). Reaction-diffusion processes and metapopulation models in heterogeneous networks. Nature Physics, 3, 276-282.

Coyne, R., Barton, N. H., \& Turelli, M. (1997). Perspective: A critique of Sewall Wright's shifting balance theory of evolution. Evolution, 51, 643-671.

Craver, C. (2007). Explaining the brain. New-York: Oxford University Press.

Craver, C. (2013). Functions and mechanisms: A perspectivalist account. In P. Huneman (Ed.), Functions: Selection and mechanism (pp. 133-158). Dordrecht: Springer.

Craver, C., \& Bechtel, W. (2007). Top-down causation without top-down causes. Biology and Philosophy, 22, 547-563.

Craver, C., \& Darden, L. (2013). In search for mechanisms: Discovery across the life sciences. Chicago: University of Chicago Press.

Davidson, E., McClay, D., \& Hood, L. (2003). Regulatory gene networks and the properties of the developmental process. PNAS, 100, 1475-1480.

Davidson, E. H. (1986). Gene activity in early development. Orlando: Academic Press.

Dorato, M., \& Felline. L. (2011). Scientific explanation and scientific structuralism. In A. Bokulich \& P. Bokulich (Eds.), Scientific structuralism, Boston studies in the philosophy of science (pp. 161-177). Springer.

de la Fuente, A. (2009). What are gene regulatory networks? In S. Das, D. Caragea, S. Welch, \& W. H. Hsu (Eds.), Handbook of research on computational methodologies in gene regulatory networks (pp. 1-27). Hershey, PA: Medical Information Science Reference.

Elton, C. S. (1927). Animal ecology. London: Sidgwick and Jackson.

Fernandez, P., \& Solé, R. (2005). Neutral fitness landscapes in signalling networks. Journal of The Royal Society Interface, 4(12), 41-47.

Fontaine, C., Guimaraes, P., Kéfi, S., Loeuille, N., Memmott, J., van der Putten, W. H., et al. (2011). The ecological and evolutionary implications of merging different types of networks. Ecology Letters, 14(11), 1170-1181.

Frank, S. A. (2009). The common patterns of nature. Journal of Evolutionary Biology, 22, 1563-1585. 
Gavrilets, S. (1999). A dynamical theory of speciation on holey adaptive landscapes. American Naturalist, $154,1-22$.

Gavrilets, S. (2003). Evolution and speciation in a hyperspace: The roles of neutrality, selection, mutation and random drift. In J. Crutchfield \& P. Schuster (Eds.), Towards a comprehensive dynamics of evolution: Exploring the interplay of selection, neutrality, accident, and function (pp. 135-162). New-York: Oxford University Press.

Gayon, J. (1998). Darwinism's struggle for survival: heredity and the hypothesis of natural selection. Cambridge: Cambridge University Press.

Gilbert, S. F., Opitz, G., \& Raff, R. (1996). Resynthesizing evolutionary and developmental biology. Developmental Biology, 173, 357-372.

Glennan, S. (1996). Mechanisms and the nature of causation. Erkenntnis, 44, 49-71.

Grafen, A. (2007). The formal Darwinism project: A mid-term report. Journal of Evolutionary Biology, 20, $1243-1254$.

Granovetter, M. (1973). The strength of weak ties. American Journal of Sociology, 78(6), 1360-1380.

Gross, J. L., \& Tucker, T. W. (1987). Topological graph theory. Reading: Wiley Interscience.

Hanski, I. (1998). Metapopulation dynamics. Nature, 396, 41-49.

Huneman, P. (2010a). Topological explanations and robustness in biological sciences. Synthese, 177(2), 213-245.

Huneman, P. (2010b). Assessing the prospects for a return of organisms in evolutionary biology. History and Philosophy of the Life Sciences, 32, 341-372.

Huneman, P. (2014). A pluralist framework to address challenges to the modern synthesis in evolutionary theory. Biological Theory, 9(2), 163-177.

Huth, G., Pittard, E., Haegemann, B., \& Munoz, F. (2015). Long-distance rescue and slow extinction dynamics govern multiscale metapopulations. American Naturalist, (In press).

Jones, N. (2014). Bowtie structures, pathway diagrams, and topological explanation. Erkenntnis, 79(5), $1135-1155$

Justus, J. (2008). Complexity, diversity, and stability. In S. Sarkar \& A. Plutynski (Eds.), Companion to the philosophy of biology (pp. 321-350). London: Blackwell.

Kaplan, D., \& Craver, C. (2011). The explanatory force of dynamical and mathematical models in neuroscience: A mechanistic perspective. Philosophy of Science, 78(4), 601-627.

Kauffmann, S. (1993). Origins of order: Self-organization and selection in evolution. Oxford: Oxford University Press.

Kimura, M. (1983). The neutral theory of molecular evolution. Cambridge: Cambridge University Press.

Kitano, H., \& Oda, K. (2006). Robustness trade-offs and host-microbial symbiosis in the immune system. Molecular Systems Biology, 2, 1-10.

Klemm, K., \& Bornholdt, S. (2005). Topology of biological networks and reliability of information processing. PNAS, 102, 18414.

Lamm, E. (2014). The genome as a developmental organ. Journal of Physiology, 592(11), 2237-2244.

Lesne, A. (2007). Complex networks: From graph theory to biology. Letters in Mathematical Physics, 78, $235-262$.

Lewontin, R. (1974). The genetic basis of evolutionary change. New York: Columbia University Press.

Lynch, M. (2007). The origins of genome architecture. Boston: Sinauer.

Lyon, A. (2014). Why are normal distributions normal? The British Journal for the Philosophy of Science, 65(3), 621-649. doi:10.1093/bjps/axs046.

Matthews, P., et al. (2011). Toward an integration of evolutionary biology and ecosystem science. Ecology Letters, 14, 690-701.

May, R., Levin, S., \& Sugihara, G. (2008). Complex systems: Ecology for bankers. Nature, 451, 893-895.

Mayr, E., \& Provine, W. (1980). The evolutionary synthesis. Perspectives on the unification of biology. Cambridge: Harvard University Press.

Monod, J., Wyman, J., \& Changeux, J. P. (1965). On the nature of allosteric transitions: A plausible model. Journal of Molecular Biology, 12, 88-118.

Montoya, J., Pimm, S., \& Solé, R. (2006). Ecological networks and their fragility. Nature, 442, 259-267.

Olff, H., Alonso, D., Berg, M. P., Eriksson, B. K., Loreau, M., Piersma, T., et al. (2009). Parallel ecological networks in ecosystems. Philosophical Transactions of the Royal Society of London B: Biological Sciences, 364, 1755-1779.

Orr, H. A. (2009). Fitness and its role in evolutionary genetics. Nature Reviews Genetics, 10, 531-539.

Pimm, S. (1985 [2002]). Food webs ( $2^{\text {nd }}$ ed.). Chicago: University of Chicago Press. 
Post, D., \& Palkovacs, E. (2009). Eco-evolutionary feedbacks in community and ecosystem ecology: Interactions between the ecological theatre and the evolutionary play. Philosophical Transactions of the Royal Society B: Biological Sciences, 364, 1629-1640.

Potochnik, A. (2009). Optimality modelling in a suboptimal world. Biology and Philosophy, 24(2), $183-197$.

Raff, R. (1996). The shape of life. Chicago: University of Chicago Press.

Rice, C. (2012). Optimality explanations: A plea for an alternative approach. Biology and Philosophy, 27(5), 685-703.

Rodriguez Caso, C., \& Conde-Puyeo, N. (2009). Topological analysis of cellular networks. In E. G. Giannopoulou (Ed.), Data mining in medical and biological research (pp. 253-267). Vienna: ARS publishing.

Rose, M., \& Lauder, G. (1996). Adaptation. San Diego: Academic Press.

Seung, S. H. (2009). Neuroscience: Towards functional connectomics. Nature, 471, 170-172.

Short, M., Brantingham, J., Bertozzi, A., \& Titad, G. (2010). Dissipation and displacement of hotspots in reaction-diffusion models of crime. PNAS, 107(9), 3961-3965.

Skipper, R., \& Millstein, R. (2005). Thinking about evolutionary mechanisms: Natural selection. Studies in History and Philosophy of Biological and Biomedical Sciences, 36 604(2), 327-347.

Solé, R. V., \& Montoya, J. M. (2001). Complexity and fragility in ecological networks. Proceedings of the Royal Society of London B: Biological Sciences, 268, 2039-2045.

Solé, R., \& Valverde, S. (2006). Are network motifs the spandrels of cellular complexity? TREE, 21(8), 419-22.

Sporns, O. (2012). Discovering the human connectome. Cambridge: MIT Press.

Strogatz, S. (2001). Exploring complex networks. Nature, 410, 268-276.

Turing, A. (1952). The chemical basis of morphogenesis. Philosophical Transactions of the Royal Society of London. Series B, 237(641), 37-72.

Ulanowicz, R. (2011). Quantitative methods for ecological network analysis and its application to coastal ecosystems. Treatise on Estuarine and Coastal Science, 9, 35-57.

Van Gelder, T. (1995). What might cognition be, if not computation? Journal of Philosophy, 92(7), 345-381.

Wimsatt, W. (1972). Complexity and organization. PSA: Proceedings of the Biennial Meeting of the Philosophy of Science Association, 6, 67-85.

Woodward, J. (2013). Mechanistic explanation: Its scope and limits. Aristotelian Society Supplementary Volume, 87(1), 39-65.

Wright, S. (1931). Evolution in mendelian populations. Genetics, 16, 97-159.

Yokoyama, S. (2000). Molecular evolution of vertebrate visual pigments. Progress in Retinal and Eye Research, 19(4), 385-419. 